\title{
Health risks of frozen raw breaded chicken products: A local health unit perspective
}

\author{
Julia Pinheiro Carvalho, Mike Benusic ${ }^{2}$, Raluca Codat ${ }^{3}$, Roxana Gruescu³, Liz Haydu³, \\ Lawrence C Loh ${ }^{2,3 *}$
}

\begin{abstract}
Raw chicken, including frozen raw breaded chicken products, has been implicated in 18 outbreaks of nontyphoidal salmonellae in Canada since 2017. The Canadian Food and Inspection Agency has since implemented industry requirements, from April 2019, aimed at reducing salmonellosis risks in frozen raw breaded chicken products prior to their distribution in the retail marketplace. This commentary explores key issues identified by a local public health unit during an investigation of two cases of salmonellosis that occurred within the context of a recent Canada-wide outbreak linked to frozen raw breaded chicken products. Consumer handling and preparation practices, product appearance and labelling issues were essential factors in the development of disease. From this front-line perspective, new industry requirements by the Canadian Food and Inspection Agency are analyzed for their potential to reduce salmonellosis risks in such chicken products, while also identifying additional measures that could be implemented to further reduce the risk of product associated outbreaks.
\end{abstract}

Suggested citation: Pinheiro Carvalho J, Benusic MA, Codat R, Gruescu R, Haydu L, Loh LC. Health risks of frozen raw breaded chicken products: A local health unit perspective. Can Commun Dis Rep 2020;46(11/12):403-8. https://doi.org/10.14745/ccdr.v46i1112a07

Keywords: salmonella, raw chicken, food safety, requirements

\section{Introduction}

Nontyphoidal salmonellae infection remains a leading cause of foodborne illness in Canada with an annual average of 6,881 reported cases from 2008 to 2017, which represents an average rate of 20 cases per 100,000 people per year (1). In 2018, the national incidence of salmonellae infections was 19 cases per 100,000 people, a significant decrease compared to 2017 , while Ontario registered a significant increase from 12 to 18 cases per 100,000 people in the same period. Salmonella Enteritidis is the most commonly reported serovar for Canadians, involved in $43 \%$ of cases, followed by Salmonella Heidelberg (9\%) and Typhimurium (6\%) (2).

Severity of salmonellae infections (also referred to as salmonellosis) may vary, and while most cases resolve without treatment, others might result in severe dehydration, bacteremia and death, particularly among vulnerable populations such as young children, the elderly, pregnant women, and those who are immunocompromised (3). Salmonellosis' burden includes an estimated $\$ 6.43$ million annually in healthcare costs and $\$ 21.13$ million annually in productivity loss (4).

Many commercial food products contaminated with salmonellae have been implicated in outbreaks, including meat, eggs, dairy, fruits and vegetables (3). Of particular interest in recent times are frozen raw breaded chicken products, such as chicken strips and chicken nuggets, which have been linked to salmonellae outbreaks in the United States (5), Australia (6) and Canada since the 1990s (7). Since then, studies have implicated these products as leading risk factors for salmonellosis (7-10), and data from FoodNet, a sentinel site surveillance system led by the Public Health Agency of Canada (PHAC), indicates that in 2018, salmonellae were found in $27 \%$ of samples of frozen raw breaded chicken products across its sites (2).

In May 2017, whole genome sequencing was introduced for analysis of all clinical Salmonella isolates in Canada, providing increased resolution of genetic relatedness among Salmonella isolates and facilitating the identification of clusters and outbreaks of common Salmonella serotypes such as Enteritidis (11). The implementation of whole genome sequencing has permitted linking of 18 salmonellosis outbreaks across the country between May 2017 and Mary 2019 to the consumption of chicken products, amounting to 584 laboratory-confirmed cases, 97 hospitalizations. This led to the removal of 14 such products from the marketplace, 13 of which being voluntarily removed by the manufacturers from the marketplace following food recalls issued by the Canadian Food and Inspection Agency (CFIA) (12). Of these, 12 outbreaks and 
285 cases were found to be directly associated with exposure to frozen raw breaded chicken products (11). The Region of Peel in Ontario saw similar trends in 2016 and 2017, with annual reports of at least 30 children aged nine or younger developing salmonellosis after consuming these products; a number that likely suffers from some degree of underreporting (13).

In response to these outbreaks, the CFIA announced in July 2018 new requirements for industry to implement manufacturing or processing measures reducing salmonellae to undetectable levels in these products before distribution that took effect on April 1, 2019 (14). This commentary offers a local public health perspective on the risks associated with consumption of raw breaded chicken products by describing contributing factors in two cases of salmonellosis investigated by Peel Public Health and discusses how these might be addressed by the new CFIA requirements.

\section{A local health unit outbreak investigation}

In summer 2018, Peel Public Health was notified by local laboratories of two different cases of salmonellosis identified through stool testing, following the usual notification cascade in Ontario. Each case was assigned to a public health inspector in charge of conducting interviews and collecting any necessary samples to help establish the source of infection. Case 1 was a three-year-old female and Case 2 an eleven-year-old male. Each case separately presented at emergency with fever and diarrhea developing over a period of five days and each provided clinical samples for bacterial culture; while both were subsequently discharged, Case 2 was later admitted to hospital for intravenous antibiotic therapy (see Table 1 for case comparison overview).

Table 1: Comparison overview of confirmed cases investigated by Peel Public Health agents in the context of a salmonellosis outbreak in the summer of 2018

\begin{tabular}{|c|c|c|}
\hline $\begin{array}{l}\text { Characteristics } \\
\text { investigated }\end{array}$ & Case 1 & Case 2 \\
\hline Sex & Female & Male \\
\hline Age & 3 years & 11 years \\
\hline $\begin{array}{l}\text { Hospitalization } \\
\text { required }\end{array}$ & No & Yes \\
\hline $\begin{array}{l}\text { Method of } \\
\text { preparation of } \\
\text { implicated frozen } \\
\text { chicken product }\end{array}$ & Oven baking & Pan-frying $^{a}$ \\
\hline
\end{tabular}

For Case 1, a food diary and investigation revealed the consumption of a nationally distributed brand of frozen raw breaded chicken nuggets and another brand of pre-cooked chicken strips within the illness incubation period. Both food products had been prepared by the case's mother as per the instructions listed on the package (see Box 1 for chicken nuggets cooking instructions). No one else in the household consumed the food or became ill. Samples from the frozen raw breaded chicken nuggets meal collected from the case home by public health inspectors and submitted to the Ontario Public Health Laboratory for testing and were positive for Salmonella Enteritidis; testing of samples from the pre-cooked chicken strips meal were negative. Subsequent whole genome sequencing matched the isolates from the case's stool sample to those from the frozen raw breaded chicken nuggets.

Box 1: Instructions on the inner package of frozen raw breaded chicken nuggets implicated in the outbreak

Do not microwave.

Raw Poultry.

Must be cooked thoroughly.

\section{Conventional Oven:}

1. Preheat oven to $425^{\circ} \mathrm{F}\left(220^{\circ} \mathrm{C}\right)$.

2. Place breaded chicken on a non-stick or lightly greased baking sheet.

3. Cook on the middle oven rack according to the times below:

Nuggets: Cook 8 minutes then turn and continue cooking for another 5-7minutes.

Individual appliances vary; these are guidelines only. Cook thoroughly to an internal temperature of $165^{\circ} \mathrm{F}\left(74^{\circ} \mathrm{C}\right)$.

Handling instructions: ensure that raw meat and poultry products are handled and cooked properly. Keep frozen until ready to prepare. Keep cooked/ready-to-eat foods separate from raw foods. Refrigerate leftovers immediately. Thoroughly wash working surfaces, utensils, and hands after touching raw meat and poultry.

${ }^{a}$ Identical instructions in French were printed next to the English ones

For Case 2, a food diary and investigation found that the eleven-year old male consumed the same brand of frozen raw breaded chicken nuggets as Case 1, within the same incubation period. These were not prepared according to the package instructions as they were pan-fried before consumption (see Box 1). No one else in the household consumed the food or became ill. Testing of samples from the frozen raw breaded chicken nuggets meal were positive for Salmonella Enteritidis. 
On whole genome sequencing, these isolates from the frozen raw breaded chicken nuggets sample matched those found in Case 1. Samples of the same brand of breaded chicken nuggets consumed by confirmed cases in Alberta and British Columbia provided additional genomic sequencing matches of Salmonella Enteritidis, and resulted in the product manufacturer issuing a food recall of the implicated food product (15).

\section{Discussion}

\section{Key issues identified at the local public health level}

Through review of these two cases and other previous investigations, our health unit identified four key issues that likely contributed to salmonellae transmission through frozen breaded chicken products. First, as Case 1 illustrated, was the risk of salmonellae contamination despite product preparation as per the package directions. This could have been due to a breach of food safety practices, such as not handwashing before and after handling the raw breaded chicken products and overlooking the need to sanitize surfaces, dishes and utensils after food preparation and consumption.

Second was the product appearance. These products have been par-fried by the manufacturer, which produces a brown colour on the outside of breaded chicken products. This may lead consumers to believe the food is cooked $(16,17)$ and subsequently undercook it, using alternative preparation methods such as microwaving or pan-frying. These methods may only defrost or unevenly heat the product, without allowing the raw chicken to fully cook to the recommended internal temperature of $74^{\circ} \mathrm{C}(10)$. The appearance of par-fried products may be particularly misleading to young children, with at least one previous salmonellosis investigation by this local public health unit that arose from a child biting into a frozen chicken product.

Third, inspection by public health investigators revealed the warning labels of the implicated products were small and difficult to read. Fourth, the lot code printed on the inner plastic liner was stamped over the cooking instructions, obscuring both the lot code and the instructions. These aspects of information provided to consumers is particularly relevant in light of previous observations by the health unit that consumers report discarding the box and storing the nuggets in the freezer in the plastic liner only. If the preparation instructions are only printed on the box, they are no longer accessible to the consumer when the box is discarded. Consumers have reported in previous outbreak investigations discarding the box because it is bulky and takes up too much space in the freezer.
Some or all of these four factors may have contributed to Case 2, where the investigation found that the nuggets were prepared incorrectly through pan-frying. While a specific reason for ignoring the instructions was not determined at the time of the investigation, one can hypothesize that clearer instructions, which are not obscured and are printed directly on the inner package, may increase the likelihood that the product will be correctly prepared.

\section{New control measures}

Effective April 1, 2019, the CFIA implemented new requirements for manufacturers to control the risk of salmonellae in frozen processed raw chicken products (13). Options for control measures consist of either inclusion of a validated cooking process, implementation of a testing program, a combination of both, or a hold-and-test program for finished products (18). These requirements target specifically non-intact raw, breaded, par-fried chicken products for retail sale, as they are at higher risk for both contamination through processing and undercooking because of their appearance (18). Notably, these requirements do not apply to products for sale to food service processors nor breaded par-fried stuffed chicken products. While the requirements aim to address the root cause of these outbreaks, i.e. exposure to salmonellae in non-stuffed frozen raw breaded chicken products for retail sale, they still leave some residual transmission risk through other products and through the food service industry. Residual risk also remains as the ready-to-eat appearance of par-fried stuffed chicken products might continue to result in unsafe product preparation or handling.

Other key contributing issues identified in our health unit review are related to product labelling and packaging, and fall outside the scope of new CFIA requirements. Mandatory labelling measures put in place in 2004 required manufacturers to put descriptors such as "uncooked" to be placed near the product's name and for cooking instructions to appear on the outer packaging (17). Furthermore, the CFIA, Health Canada and PHAC worked with industry in 2015 to develop voluntary labelling strategies with instructions to ensure consistent messaging, explicit warnings against microwaving the products, and to provide cooking directions on the inner packaging (17). However, our investigation found that the font size used for the "uncooked" label was very small and, in the case of the contaminated uncooked chicken nuggets, was also obscured.

\section{Public awareness campaigns}

Although the new requirements are a critical component of a food safety strategy to address the risk of salmonellae infection from inadequately cooked breaded chicken products, consumer awareness is equally vital. Measures to address this are already well underway at the federal level, where CFIA, Health Canada, and PHAC developed large communications campaigns to reach Canadians through social media ads and posts, outreach to 
other media and partners, and overarching and outbreak-specific public health notices (17). Additionally, in September 2018, the outbreaks prompted Canada's Council of Chief Medical Officers of Health to issue a statement (see Box 2) advising consumers of the importance of following safe food handling and preparation practices when consuming frozen raw breaded chicken products (19).

Local health units have also made efforts to improve consumer awareness of the issue through public communication. For example, in 2018, Peel Public Health ran a local education campaign in Peel Region raising awareness of the outbreaks and the various hazards of consuming undercooked frozen raw breaded chicken products. This campaign consisted of targeted Google ads, editorials in three local newspapers, and web and social media information on safe food preparation practices (see Figure 1). The local health unit has also since begun to review evidence to determine effective interventions to encourage safe food handling by consumers at home, with the goal of reducing the risk of foodborne illness arising from risky food handling practices in the home setting.

\section{Future challenges}

Much has been done to address the risk of salmonellosis in frozen breaded chicken products that has included industry-level preventive measures and multi-stakeholder efforts to increase public awareness of the issue. However, as was demonstrated by the outbreak linked to frozen raw breaded chicken products that were sold until May 2019 (12) and therefore after the new CFIA requirements came into effect, salmonellosis outbreaks associated with frozen raw breaded chicken products may not yet be a phenomenon of the past. It may well be that this outbreak was a part of a transitional period. Since products produced prior to April 1, 2019 could still be available for up to two years in the marketplace or in consumers' freezers (16), occasional exposure is still possible, but occurrence of new cases should decrease in the coming years. Cases may also emerge in association with products unaffected by the new requirements, such as stuffed chicken products.
Box 2: Excerpt of September 13, 2018 statement: Council of Chief Medical Officers of Health concerned about the risk of salmonellosis from frozen raw breaded chicken products (19)

Most frozen breaded chicken products available for sale in grocery stores in Canada contain raw chicken that can cause Salmonella illness and therefore pose an increased health risk to Canadians who handle, prepare or consume them... Canadians need to be aware that even though these products may appear to be cooked, they are not.

We are very pleased that the Government of Canada is working with the food manufacturing industry and food retailers to reduce Salmonella in frozen raw breaded chicken products produced on or after April 1, 2019, to below detectable amounts...

However, until April 1, 2019, and likely for up to a year after this date, frozen raw breaded chicken products containing Salmonella will continue to be in the marketplace and in freezers across the country. This is why, collectively, we are stressing the importance of handling and preparing frozen raw breaded chicken products with caution.

Always cook your frozen raw breaded chicken products thoroughly according to the package instructions to an internal temperature of at least $74^{\circ} \mathrm{C}\left(165^{\circ} \mathrm{F}\right)$ using a digital food thermometer to ensure that they are safe to eat. Wash your hands before and after handling these products, and wash and sanitize the surfaces, dishes and utensils used to prepare and serve them. Following this advice when handling, cooking or eating these products will help reduce you and your family's chance of becoming infected with Salmonella. For more tips and information on how to properly prepare and cook frozen raw breaded chicken products, visit Canada.ca/foodsafety.

Figure 1: Messaging used in newspaper and digital advertising to highlight the health risks associated with frozen raw breaded chicken products

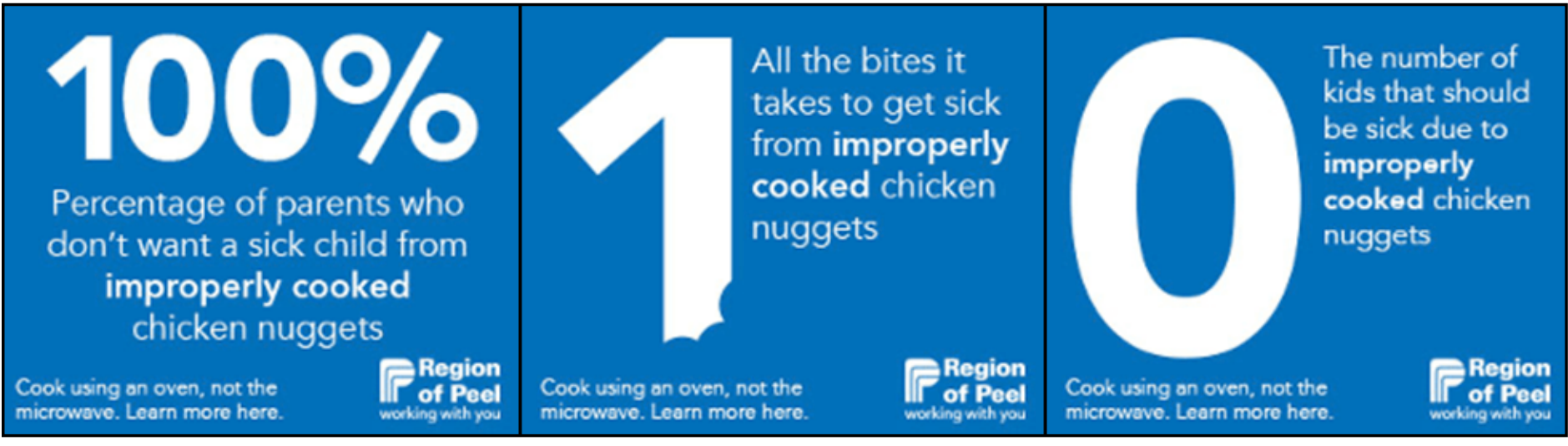


Indeed, local public health needs to continue surveillance of foodborne illness, remaining alert to whether the measures already taken will ensure the end of these outbreaks or whether further cases will still emerge. Local health units also have a role in conducting detailed case investigations and identifying emerging risk factors, which can in turn be included in future investigation forms and documents, updated regularly where policies change. Peel Public Health is also conducting research on how best to inform consumers about food safety risks in the home, and additional public awareness measures must continue to be taken by local, provincial/territorial and federal health authorities in order to increase the uptake of food safety measures by consumers. Industry-level requirements for easy-to-read labelling and ensuring unobstructed information is provided on both inner and outer packaging could complement public awareness efforts and facilitate appropriate product preparation by consumers. While a combination of modification of labels and public awareness measures alone have reportedly not been sufficient to prevent outbreaks related to frozen breaded chicken products (20), the efficacy of these measures in the presence of CFIA's new industry requirements remains to be seen.

If needed, there is room for additional work to further reduce the risk. While changing the appearance of breaded chicken products so that they are pre-cooked or do not appear to be cooked is one control mechanism, other opportunities for protection that did not emerge through our investigations but have been identified in other Canadian outbreaks involve product packaging and marketing. For example, placement of uncooked products next to fully cooked ones in grocery store freezers and packaging showing the cooked product and raw products being marketed as quick and easy meals may be misleading to consumers (7) and present other potential avenues for risk reduction through regulation by health authorities.

\section{Conclusion}

Canada's salmonellosis outbreaks from frozen breaded chicken products are being addressed by new industry-level requirements to reduce the risk of salmonellae contamination of these products for retail purchase as well as public awareness campaigns from the local to federal levels. Local public health units are essential partners and continue to be engaged in this issue from the perspective of local surveillance, investigation, evidence collection and collaboration with other agencies such as Public Health Ontario and the CFIA. Research on how best to inform consumers about food safety risks in the home is also being conducted.

\section{Authors' statement}

$L C L, J P C$ and MB jointly conceived the idea for this manuscript. JPC developed the first draft. All authors contributed to the development and revision of this manuscript and approved the final draft for submission.

\section{Competing interests}

None.

\section{Acknowledgements}

We thank L Aubin, P Callanan, A Chiefari, and J LeMoine for their contributions to the drafting of this manuscript, as well as Ms. A Hexemer from the Public Health Agency of Canada for her feedback and assistance in the revision of this manuscript.

\section{Funding}

None received

\section{References}

1. Public Health Agency of Canada. Reported cases from 1924 to 2017 in Canada - Notifiable diseases on-line. Ottawa (ON): PHAC (updated 2020-01-15; accessed 2020-06-29). http://dsol-smed.phac-aspc.gc.ca/notifiable/charts?c=pl

2. Public Health Agency of Canada. FoodNet Canada annual report 2018. Salmonella. Ottawa (ON): PHAC; Mar 28, 2019 (accessed 2020-09-08). https://www.canada.ca/ en/public-health/services/surveillance/foodnet-canada/ publications/foodnet-canada-annual-report-2018. html\#salmonella-2.1

3. Behravesh CB, Griffin PM. Salmonellosis. In: Heymann DL, editor. Control of Communicable Diseases Manual. American Public Health Association; 2015. pp. 1-2. https://ccdm. aphapublications.org/doi/book/10.2105/CCDM.2745

4. Jain S, Mukhopadhyay K, Thomassin PJ. An economic analysis of salmonella detection in fresh produce, poultry, and eggs using whole genome sequencing technology in Canada. Food Res Int. 2019;116:802-9. DOI

5. US Centres for Disease Control and Prevention. Multistate Outbreak of Drug-Resistant Salmonella Enteritidis Infections Linked to Raw, Frozen, Stuffed Chicken Entrees Produced by Barber Foods (Final Update). Washington (DC) (updated 2015-10-16; accessed 2020-06-29). https://www.cdc.gov/ salmonella/frozen-chicken-entrees-07-15/

6. Kenny B, Hall R, Cameron S. Consumer attitudes and behaviours--key risk factors in an outbreak of Salmonella typhimurium phage type 12 infection sourced to chicken nuggets. Aust N Z J Public Health 1999;23(2):164-7. DOI PubMed

7. Hobbs JL, Warshawsky B, Maki A, Zittermann S, Murphy A, Majury A, Middleton D. Nuggets of Wisdom: Salmonella Enteritidis Outbreaks and the Case for New Rules on Uncooked Frozen Processed Chicken. J Food Prot 2017;80(4):703-9. DOI PubMed 
8. Currie A, MacDougall L, Aramini J, Gaulin C, Ahmed R, Isaacs $S$. Frozen chicken nuggets and strips and eggs are leading risk factors for Salmonella Heidelberg infections in Canada. Epidemiol Infect 2005;133(5):809-16. DOI PubMed

9. MacDougall L, Fyfe M, McIntyre L, Paccagnella A, Cordner K, Kerr A, Aramini J. Frozen chicken nuggets and strips--a newly identified risk factor for Salmonella Heidelberg infection in British Columbia, Canada. J Food Prot 2004;67(6):1111-5. DOl PubMed

10. Middleton $D$, Savage $R$, Tighe MK, Vrbova L, Walton $R$, Whitfield Y, Varga C, Lee B, Rosella L, Dhar B, Johnson C, Ahmed R, Allen VG, Crowcroft NS. Risk factors for sporadic domestically acquired Salmonella serovar Enteritidis infections: a case-control study in Ontario, Canada, 2011. Epidemiol Infect 2014;142(7):1411-21. DOI PubMed

11. Morton VK, Kearney A, Coleman S, Viswanathan $M$, Chau K, Orr A, Hexemer A. Outbreaks of Salmonella illness associated with frozen raw breaded chicken products in Canada, 2015-2019. Epidemiol Infect 2019;147:e254. DOI PubMed

12. Public Health Agency of Canada. Public Health Notice Outbreaks of Salmonella infections linked to raw chicken, including frozen raw breaded chicken products. Ottawa (ON): PHAC (updated 2019-05-25; accessed 2020-06-29). https://www.canada.ca/en/public-health/ services/public-health-notices/2018/outbreaks-salmonell a-infections-linked-raw-chicken-including-frozen-raw-bre aded-chicken-products.html

13. Peel Public Health. Preparing Frozen Store-Bought Chicken Nuggets. Region of Peel (ON): Peel Public Health (updated 2018-11-05; accessed 2020-09-14). https://www.peelregion. $\mathrm{ca} /$ health/frozenchicken/index.htm

14. Canada Food Inspection Agency. Notice to Industry New requirements to reduce Salmonella to below detectable amounts in frozen raw breaded chicken products. Ottawa (ON): CFIA (updated 2019-04-01; accessed 2020-09-08). https://www.inspection.gc.ca/ food-safety-for-industry/archived-food-guidance/ meat-and-poultry-products/program-changes/2018-07-12/ eng/1520884138067/1520884138707
15. Public Health Agency of Canada. Canada's Foodborne Illness Outbreak Response Protocol (FIORP): A guide to multi-jurisdictional enteric outbreak response. Ottawa (ON): PHAC (updated 2018-02-21; accessed 2020-09-08). https://www.canada.ca/en/public-health/services/ publications/health-risks-safety/canadas-foodborn e-illness-outbreak-response-protocol-fiorp-guidemulti-jurisdictional-enteric-outbreak-response.html

16. Health Canada. Frozen raw breaded chicken. Ottawa (ON): $\mathrm{HC}$; updated HC (updated 2019-02-06; accessed 202006-29). https://www.canada.ca/en/health-canada/services/ meat-poultry-fish-seafood-safety/frozen-breaded-chicken. html

17. Canadian Food Inspection Agency. Questions and answers: New measures to reduce salmonella in frozen raw breaded chicken products. Ottawa (ON): CFIA (updated 2019-04-01; accessed 2020-06-29). https://www.inspection.gc.ca/preventive-controls/ meat/salmonella-in-frozen-raw-breaded-chicken/faq/ eng/1554140834819/1554140994648

18. Canadian Food Inspection Agency. Salmonella control options in frozen raw breaded chicken products. Ottawa (ON): Government of Canada (updated 2019-04-01; accessed 2020-06-29). http://inspection.gc.ca/food/ general-food-requirements-and-guidance/preventive-control s-food-businesses/meat/salmonella-in-frozen-ra w-breaded-chicken/eng/1531254524193/1531254524999

19. Public Health Agency of Canada. Council of Chief Medical Officers of Health concerned about the risk of Salmonella illness from frozen raw breaded chicken products. Ottawa (ON): PHAC (updated 2018-09-14; accessed 2020-06-29). https://www.canada.ca/en/public-health/news/2018/09/ council-of-chief-medical-officers-of-health-concerned-a bout-the-risk-of-salmonella-illness-from-frozen-raw-breadedchicken-products.html

20. Smith KE, Medus C, Meyer SD, Boxrud DJ, Leano F, Hedberg CW, Elfering K, Braymen C, Bender JB, Danila RN. Outbreaks of salmonellosis in Minnesota (1998 through 2006) associated with frozen, microwaveable, breaded, stuffed chicken products. J Food Prot 2008;71(10):2153-60. DOI PubMed 